Controllability and observability of the photophysical system of intermolecular two-state excited-state processes

Non Peer-reviewed author version

Boens, N; Novikov, E \& AMELOOT, Marcel (2008) Controllability and observability of the photophysical system of intermolecular two-state excited-state processes. In: JOURNAL OF PHYSICAL CHEMISTRY A, 112(12). p. 2738-2742.

DOI: $10.1021 / \mathrm{jp} 710050 \mathrm{e}$

Handle: http://hdl.handle.net/1942/8185 


\title{
Controllability and Observability of the Photophysical System of Intermolecular Two-State Excited-State Processes
}

REVISED: January 10, 2008

\author{
Noël Boens, , ,a Eugene Novikov, ${ }^{\mathrm{b}}$ Marcel Ameloot ${ }^{\mathrm{c}}$ \\ ${ }^{a}$ Department of Chemistry, Katholieke Universiteit Leuven, Celestijnenlaan 200f-bus \\ 02404, 3001 Heverlee (Leuven), Belgium \\ ${ }^{b}$ Institut Curie, Service Bioinformatique, 26 Rue d'Ulm, Paris Cedex 05, 75248 France \\ ${ }^{c}$ Biomedical Research Institute, Hasselt University and transnationale Universiteit \\ Limburg, Agoralaan, Building D, 3590 Diepenbeek, Belgium
}

\begin{abstract}
In this report, we focus on the consequences of controllability and observability on the number of distinct exponential terms in the fluorescence decay and on the identifiability analysis of the photophysical model of intermolecular two-state excited-state processes. Controllability and observability prove to be useful concepts in photophysics for exploring methodically the conditions under which intermolecular two-state excited-state processes lead to single-exponential fluorescence $\delta$-response functions. A detailed discussion on the distinction of the possible origins of mono-exponential fluorescence decays is presented. We also show that the similarity transformation approach to identifiability leads to erroneous conclusions concerning which model parameters can be identified if this photophysical system is not controllable or not observable. The results obtained for this relatively simple photophysical system can be extended in a systematic way to more complicated photophysical models.
\end{abstract}

\footnotetext{
* To whom correspondence should be addressed. E-mail: Noel.Boens@chem.kuleuven.be. Fax: +32-16-327-990
} 


\section{Introduction}

Time-resolved fluorescence is a valuable tool available to the photophysicist for investigating the dynamics of excited-state processes. ${ }^{1,2,3}$ When a specific model is proposed to describe the kinetics of fluorescence relaxation, initially one should investigate if the underlying parameters defining the model can be determined unambiguously from error-free fluorescence decay data. This is the topic of the deterministic (or a priori) identifiability (or identification) analysis. ${ }^{4,5,6,7}$ The term $a$ priori indicates that the analysis can (and should) be done before a proposed experiment is carried out. Identifiability is of great practical importance because it tells one which information is theoretically accessible from the fluorescence decay surface and therefore, it allows one to evaluate if the parameter estimation can succeed at all.

Since the first identification analysis of an intermolecular two-state excited-state process, ${ }^{8}$ identifiability studies of a broad range of photophysical models of intermolecular as well as intramolecular two-state and three-state excited-state processes have been reported (see Refs [9] and [10] for literature data). Recently, we have described identifiability analyses of models for rotational diffusion monitored by time-

resolved fluorescence depolarization ${ }^{11,12,13,14,15}$ and of models for fluorescence quenching in aqueous micellar systems. ${ }^{16,17}$

In this report, we introduce the important concepts of controllability and observability into the field of photophysics. It will be shown that the investigated non-controllable or non-observable, intermolecular, two-state excited-state system always leads to singleexponential fluorescence $\delta$-response functions instead of the bi-exponential ones found for controllable and observable systems. Controllability and observability are useful 
concepts to investigate systematically the cases where mono-exponential fluorescence decays are observed. We will also illustrate - using the same, relatively simple, photophysical model - that controllability and observability are both required for the similarity transformation criterion to identifiability to be applicable. Indeed, the identification analysis via similarity transformation leads to erroneous results for this photophysical system when it is not controllable or not observable.

\section{Time-resolved fluorescence, controllability, and observability of the photophysical system of intermolecular two-state excited-state processes}

Consider a linear, time-invariant, dynamic, intermolecular photophysical system, consisting of two different ground-state species (labeled $\mathbf{1}$ and 2), which is photoexcited with a pulse $u(t)$ as shown in Figure 1. Photo-excitation creates, in principle, two corresponding excited species (labeled $\mathbf{1}^{*}$ and $\mathbf{2}^{*}$, respectively). As a response to that input, the concentrations $\mathbf{x}^{*}(t)$ of the excited species $\mathbf{1}^{*}$ and $\mathbf{2}^{*}$ change as a function of time $t$ and can be described by the following differential matrix equation: ${ }^{4-6,18}$

$$
\dot{\mathbf{x}}^{*}(t)=\mathbf{A} \mathbf{x}^{*}(t)+\mathbf{b}_{i} u(t), \quad \mathbf{x}^{*}\left(0^{-}\right)=0
$$

with $\dot{\mathbf{x}}^{*}(t)=\frac{d \mathbf{x}^{*}(t)}{d t}$

$$
\begin{aligned}
& \mathbf{x}^{*}(t)=\left(\begin{array}{l}
x_{1}{ }^{*}(t) \\
x_{2}{ }^{*}(t)
\end{array}\right)=\left(\begin{array}{l}
{\left[\mathbf{1}^{*}\right](t)} \\
{\left[\mathbf{2}^{*}\right](t)}
\end{array}\right) \\
& \mathbf{A}=\left(\begin{array}{ll}
a_{11} & a_{12} \\
a_{21} & a_{22}
\end{array}\right)=\left(\begin{array}{cc}
-\left(k_{01}+k_{21}[\mathrm{M}]\right) & k_{12} \\
k_{21}[\mathrm{M}] & -\left(k_{02}+k_{12}\right)
\end{array}\right)
\end{aligned}
$$


and $\mathbf{b}_{i}=\left(\begin{array}{ll}b_{1 i} & b_{2 i}\end{array}\right)^{T}$, where $T$ symbolizes transpose and the subscript $i$ refers to the excitation wavelength $\lambda_{i}^{\text {ex }}$ dependence of $\mathbf{b}$. [M] stands for the experimentally known concentration of co-reactant.

\section{$<$ Figure 1 >}

In fluorescence decay experiments, one does not follow the time course of the concentrations $\mathbf{x}^{*}(t)$ of the individual excited species, but one observes - within a selected emission band around emission wavelength $\lambda_{j}^{\mathrm{em}}-$ the composite experimental fluorescence decay $d(t)$ originating from the contributing excited species $\mathbf{1}^{*}$ and $\mathbf{2}^{*}$ :

$$
d(t)=\mathbf{c}_{j} \mathbf{x}^{*}(t)
$$

where $\mathbf{c}_{j}=\left(\begin{array}{ll}\mathrm{c}_{1 j} & \mathrm{c}_{2 j}\end{array}\right)^{19}$ and the subscript $j$ refers to the emission wavelength $\lambda_{j}^{\mathrm{em}}$ dependence of the spectral emission coefficients c.

Integration of eq 1yields $\mathbf{x}^{*}(t)^{4-6,18}$ and substitution of $\mathbf{x}^{*}(t)$ in eq 4 gives

$$
d(t)=\int_{0}^{t} f(t-s) u(s) \mathrm{d} s=\int_{0}^{t} f(s) u(t-s) \mathrm{d} s
$$

with the fluorescence $\delta$-response function $f(t)$ given by ${ }^{4-6,18}$

$$
f(t)=\mathbf{c}_{j} \mathrm{e}^{t \mathbf{A}} \mathbf{b}_{i}
$$

The triple $\left(\mathbf{A}, \mathbf{b}_{i}, \mathbf{c}_{j}\right)$ is called a realization of $f(t)$.

Now we introduce the important notions of controllability and observability ${ }^{5,7,20}$ into photophysics. The photophysical system is controllable if an input $u$ can be constructed which will bring $\mathbf{x}^{*}$ to any preselected state in a finite amount of time. ${ }^{20}$ The $2 \times 2$ controllability or reachability matrix $\mathbf{R}$ for the photophysical system of intermolecular two-state excited-state processes is defined in eq $7:{ }^{5,20}$ 


$$
\mathbf{R}=\left(\begin{array}{ll}
\mathbf{b}_{i} & \mathbf{A} \mathbf{b}_{i}
\end{array}\right)=\left(\begin{array}{ll}
b_{1 i} & a_{11} b_{1 i}+a_{12} b_{2 i} \\
b_{2 i} & a_{21} b_{1 i}+a_{22} b_{2 i}
\end{array}\right)
$$

with $a_{m n}$ defined by eq 3 . The photophysical system is controllable if and only if the associated controllability matrix $\mathbf{R}$ is of rank $2,5,7,20,21$ or, equivalently, if $\operatorname{det} \mathbf{R} \neq 0$.

$\operatorname{Rank} \mathbf{R}=2$ if $\operatorname{det} \mathbf{R}=b_{1 i}\left(a_{21} b_{1 i}+a_{22} b_{2 i}\right)-b_{2 i}\left(a_{11} b_{1 i}+a_{12} b_{2 i}\right) \neq 0$.

The following two cases reduce the rank of matrix $\mathbf{R}$ to 1 :

(a) If $b_{2 i}=0$ (i.e., ground-state species 2 does not absorb light at the excitation wavelength $\left.\lambda_{i}^{\mathrm{ex}}\right)$, then $\operatorname{det} \mathbf{R}=a_{21} b_{1 i}{ }^{2}$. To have a controllable system, $a_{21}\left(=k_{21}[\mathrm{M}]\right)$ must be different from zero. If $a_{21}=0$ (i.e., $k_{21}=0$ and/or $[\mathrm{M}]=0$ ) and $b_{2 i}=0$, excited species $\mathbf{2}^{*}$ is not formed (i.e., $\mathbf{2}^{*}$ cannot be reached, Figure $2 \mathrm{a}$ ): the system is not controllable and this results in a mono-exponential fluorescence $\delta$-response function $f(t):{ }^{10}$

$$
f(t)=b_{1 i} c_{1 j} \exp \left(-k_{01} t\right)
$$

(b) If $b_{1 i}=0$ (i.e., ground-state species $\mathbf{1}$ does not absorb light at $\lambda_{i}^{\mathrm{ex}}$ ), we have det $\mathbf{R}$ $=-a_{12} b_{2 i}{ }^{2}$. To have a controllable system, $a_{12}\left(=k_{12}\right)$ must be different from zero. If $a_{12}=$ $k_{12}=0$ and $b_{1 i}=0$, excited species $\mathbf{1}^{*}$ cannot be reached (i.e., $\mathbf{1}^{*}$ is not formed, Figure $2 \mathrm{~b}$ ) and the system is not controllable under these conditions. Then, a mono-exponential $f(t)$ is found:

$$
f(t)=b_{2 i} c_{2 j} \exp \left(-k_{02} t\right)
$$

\section{$<$ Figure 2 >}

The criterion for observability in terms of the matrices of the photophysical system is analogous to that of controllability. The current photophysical system described by eqs 1 and 4 is observable if and only if the $2 \times 2$ observability matrix $\mathbf{O}$ (eq 10) is of rank 2 , requiring that $\operatorname{det} \mathbf{O} \neq 0.5,7,20,21$ 


$$
\mathbf{O}=\left(\begin{array}{c}
\mathbf{c}_{j} \\
\mathbf{c}_{j} \mathbf{A}
\end{array}\right)=\left(\begin{array}{cc}
c_{1 j} & c_{2 j} \\
a_{11} c_{1 j}+a_{21} c_{2 j} & a_{12} c_{1 j}+a_{22} c_{2 j}
\end{array}\right)
$$

$\operatorname{Rank} \mathbf{O}=2$ if $\operatorname{det} \mathbf{O}=c_{1 j}\left(a_{12} c_{1 j}+a_{22} c_{2 j}\right)-c_{2 j}\left(a_{11} c_{1 j}+a_{21} c_{2 j}\right) \neq 0$.

The next two cases reduce the rank of matrix $\mathbf{O}$ to 1 :

(a) If $c_{2 j}=0$ (i.e., the fluorescence of only excited species $\mathbf{1}^{*}$ is being monitored at $\lambda_{j}^{\mathrm{em}}$ ), we have det $\mathbf{O}=a_{12} c_{1 j}{ }^{2}$. To have an observable system, $a_{12}$ must be different from zero. If $a_{12}=k_{12}=0$ and $c_{2 j}=0$, the photophysical system is not observable (because $\mathbf{2}^{*}$ is not observable, Figure 3a) and a single-exponential $f(t)$ is found:

$$
f(t)=b_{1 i} c_{1 j} \exp \left[-\left(k_{01}+k_{21}[\mathrm{M}]\right) t\right]
$$

(b) If $c_{1 j}=0$ (i.e., the fluorescence of only excited species $2^{*}$ is being monitored at $\lambda_{j}^{\mathrm{em}}$ ), we have det $\mathbf{O}=-a_{21} c_{2 j}{ }^{2}$. To have an observable system, $a_{21}$ must be different from zero. If $a_{21}=0$ and $c_{1 j}=0$, the photophysical system is not observable (because $\mathbf{1}^{*}$ is not observable, Figure $3 b$ ) and the mono-exponential $f(t)$ is given by: $:^{10}$

$$
f(t)=b_{2 i} c_{2 j} \exp \left[-\left(k_{02}+k_{12}\right) t\right]
$$

\section{$<$ Figure $3>$}

In the previous paragraphs, the non-controllable or non-observable systems depended exclusively on the excited-state exchange coefficients $\left(a_{21}\right.$ or $\left.a_{12}\right)$ and the parameters associated with excitation ( $b_{1 i}$ or $b_{2 i}$, for controllability) or emission $\left(c_{1 j}\right.$ or $c_{2 j}$, for observability). By looking at the form of the $\mathbf{R}$ and $\mathbf{O}$ matrices (and by creating rows or columns containing all zeros), it may appear that the cases discussed above, yielding a non-controllable system $\left[\left(a_{21}=0, b_{2 i}=0\right)\right.$ and $\left.\left(a_{12}=0, b_{1 i}=0\right)\right]$ and the parallel cases giving a non-observable system $\left[\left(a_{12}=0, c_{2 j}=0,\right)\right.$ and $\left.\left(a_{21}=0, c_{1 j}=0\right)\right]$ are the only possible cases reducing the rank of the matrices $\mathbf{R}$ and $\mathbf{O}$ to 1 . However, as the elements 
$a_{11}$ and $a_{21}$ are dependent on [M], the rank of the matrices $\mathbf{R}$ and $\mathbf{O}$ can be reduced to 1 at certain, non-zero, co-reactant concentrations [M].

The unique concentration $[\mathrm{M}]$ leading to a non-controllable system is calculated from det $\mathbf{R}=0$ and is given by

$$
[\mathrm{M}]=\frac{b_{2 i}\left\lfloor b_{1 i}\left(k_{02}+k_{12}-k_{01}\right)+b_{2 i} k_{12}\right\rfloor}{k_{21} b_{1 i}\left(b_{1 i}+b_{2 i}\right)}
$$

It is not evident if this $[\mathrm{M}]$ value is physically accessible. Indeed, it is possible that $[\mathrm{M}]$ given by eq 13 (and by eq 15 for the non-observable system, see further) has a negative value or is too high to be experimentally accessible. If [M] is given by eq $13, f(t)$ is single-exponential at all observation wavelengths $\lambda_{j}^{\mathrm{em}}$ and is expressed by

$$
f(t)=\left(b_{1 i} c_{1 j}+b_{2 i} c_{2 j}\right) \exp \left(-t \frac{b_{1 i} k_{01}+b_{2 i} k_{02}}{b_{1 i}+b_{2 i}}\right)
$$

The unique concentration $[\mathrm{M}]$ leading to a non-observable system is derived from det $\mathbf{O}$ $=0$ and is given by

$$
[\mathrm{M}]=\frac{c_{1 j}\left\lfloor-c_{1 j} k_{12}+c_{2 j}\left(k_{02}+k_{12}-k_{01}\right)\right\rfloor}{k_{21} c_{2 j}\left(c_{1 j}-c_{2 j}\right)}
$$

For this particular $[\mathrm{M}]$, the function $f(t)$ is mono-exponential at all excitation wavelengths $\lambda_{i}^{\text {ex }}$ and is specified by

$$
f(t)=\left(b_{1 i} c_{1 j}+b_{2 i} c_{2 j}\right) \exp \left(-t \frac{c_{2 j}\left(k_{02}+k_{12}\right)-c_{1 j} k_{12}}{c_{2 j}}\right)
$$

It should be noted that the mono-exponential functions $f(t)$, described by eqs 14 and 16 , have not been described before. 


\section{Identifiability via similarity transformation of the model for intermolecular two- state excited-state processes which are non-controllable or non-observable}

The true realizations $\left(\mathbf{A}, \mathbf{b}_{i}, \mathbf{c}_{j}\right)$ and the alternative realizations $\left(\overline{\mathbf{A}}, \overline{\mathbf{b}}_{i}, \overline{\mathbf{c}}_{j}\right)$ are similar if they produce the same $d(t)$ [or $f(t)]$ and are related as in eqs $17 \mathrm{a}-\mathrm{c}::^{5,20}$

$$
\begin{aligned}
& \overline{\mathbf{A}}=\mathbf{T}^{-1} \mathbf{A} \mathbf{T} \\
& \overline{\mathbf{b}}_{i}=\mathbf{T}^{-1} \mathbf{b}_{i} \\
& \overline{\mathbf{c}}_{j}=\mathbf{c}_{j} \mathbf{T}
\end{aligned}
$$

where $\mathbf{T}$ is a constant, non-singular (i.e., invertible) matrix (det $\mathbf{T} \neq 0$ ) having the same (2 $\times 2$ ) dimension as A. The matrix $\mathbf{T}$ should be independent of the experimental conditions (i.e., $\lambda_{i}^{\mathrm{ex}}, \lambda_{j}^{\mathrm{em}}$, and $\left.[\mathrm{M}]\right) .{ }^{10}$ The equivalence relations (eqs $17 \mathrm{a}-\mathrm{c}$ ) show that an alternative triple $\left(\overline{\mathbf{A}}, \overline{\mathbf{b}}_{i}, \overline{\mathbf{c}}_{j}\right)$ realizing the same $d(t)$ [or $f(t)$ ] can be found by similarity transformation. For the photophysical system considered here, $\mathbf{T}$ is given by

$$
\mathbf{T}=\left(\begin{array}{ll}
t_{1} & t_{2} \\
t_{3} & t_{4}
\end{array}\right)
$$

Now we shall show that controllability and observability are both required for the similarity transformation approach to lead to correct conclusions. It must be emphasized, however, that controllability and observability are not sufficient conditions to guarantee identifiability of the model. 


\section{A. Non-controllable system with $a_{21}=0$ and $b_{2 i}=0$}

Now we calculate the alternative $\overline{\mathbf{A}}$ (eq 17a) and $\overline{\mathbf{b}}_{i}$ (eq 17b) for the non-controllable photophysical system with $a_{21}=0$ and $b_{2 i}=0$. The matrix multiplication in eq 17a yields the following three useful equations:

$$
\begin{aligned}
& -t_{1} \bar{k}_{01}=-t_{1} k_{01}+t_{3} k_{12} \\
& t_{1} \bar{k}_{12}-t_{2}\left(\bar{k}_{02}+\bar{k}_{12}\right)=-t_{2} k_{01}+t_{4} k_{12} \\
& t_{3} \bar{k}_{12}-t_{4}\left(\bar{k}_{02}+\bar{k}_{12}\right)=-t_{4}\left(k_{02}+k_{12}\right)
\end{aligned}
$$

From the multiplication in eq $17 \mathrm{~b}$, we obtain

$$
\begin{aligned}
& t_{1} \bar{b}_{1 i}=b_{1 i} \\
& t_{3} \bar{b}_{1 i}=0
\end{aligned}
$$

From eq 20a with $b_{1 i} \neq 0$ we can conclude that $t_{1} \neq 0$ and $\bar{b}_{1 i} \neq 0$, so that from eq $20 \mathrm{~b}$ we have that $t_{3}=0$. Therefore, both $t_{1}$ and $t_{4}$ should be different from zero (otherwise, we have a singular matrix T). Equation 19a now gives $\bar{k}_{01}=k_{01}$, while eq 19c yields $\bar{k}_{02}+\bar{k}_{12}=k_{02}+k_{12}$. The alternative $\bar{b}_{1 i}$ (eq 20a) is known up to a scaling factor.

Now we consider the fluorescence $\delta$-response function $f(t)$ (eq 8 ) for this non-controllable system. The only kinetic information that can be extracted from the mono-exponential $f(t)$ $=\alpha \exp (\gamma t)$ is $\gamma=-k_{01}$. Since $f(t)$ does not include $k_{02}$ and $k_{12}$, it is impossible to identify $\left(k_{02}+k_{12}\right)$ as the identification analysis via similarity transformation wrongly indicates.

\section{B. Non-observable system with $a_{21}=0$ and $c_{1 j}=0$}

For the alternative $\overline{\mathbf{A}}$ of this non-observable photophysical system, we refer to section 3.A. The components of the alternative $\overline{\mathbf{c}}_{j}$ calculated according to eq $17 \mathrm{c}$ are 


$$
\begin{aligned}
& 0=t_{3} c_{2} \\
& \bar{c}_{2}=t_{4} c_{2}
\end{aligned}
$$

From eq 21a, we have that $t_{3}=0$, so that from eq $21 \mathrm{~b}$ we have that the alternative $\bar{c}_{2}$ is known up to a scaling factor $\left(t_{4}\right.$ must be different from zero to have an invertible $\mathbf{T}$ matrix).

The single-exponential $f(t)$ (eq 12) for this non-observable system does not contain $k_{01}$, so it is impossible to identify $k_{01}$. Also for the non-observable system, the identification via the similarity transformation technique leads to erroneous conclusions.

\section{C. Non-controllable system with $a_{12}=0$ and $b_{1 i}=0$}

Matrix multiplication in eq $17 \mathrm{a}$ for the non-controllable system with $a_{12}=0$ yields the following three informative equations:

$$
\begin{aligned}
& -t_{1}\left(\bar{k}_{01}+\bar{k}_{21}[\mathrm{M}]\right)+t_{2} \bar{k}_{21}[\mathrm{M}]=-t_{1}\left(k_{01}+k_{21}[\mathrm{M}]\right) \\
& -t_{3}\left(\bar{k}_{01}+\bar{k}_{21}[\mathrm{M}]\right)+t_{4} \bar{k}_{21}[\mathrm{M}]=t_{1} k_{21}[\mathrm{M}]-t_{3} k_{02} \\
& -t_{4} \bar{k}_{02}=t_{2} k_{21}[\mathrm{M}]-t_{4} k_{02}
\end{aligned}
$$

From eq 17b, we obtain

$$
\begin{aligned}
& t_{2} \bar{b}_{2 i}=0 \\
& t_{4} \bar{b}_{2 i}=b_{2 i}
\end{aligned}
$$

From eq $23 \mathrm{~b}$ with $b_{2 i} \neq 0$ we can conclude that $t_{4} \neq 0$ and $\bar{b}_{2 i} \neq 0$, so that from eq $23 \mathrm{a}$ we have that $t_{2}=0$. Now eq $22 \mathrm{a}$ with $t_{1} \neq 0$ (non-singular $\mathbf{T}$ matrix!) simplifies to $\bar{k}_{01}+\bar{k}_{21}[\mathrm{M}]=k_{01}+k_{21}[\mathrm{M}]$, from which we obtain $\bar{k}_{01}=k_{01}$ and $\bar{k}_{21}=k_{21}$. Equation 22c with $t_{2}=0$ gives $\bar{k}_{02}=k_{02}$. Grouping the terms in $[\mathrm{M}]$ in eq $22 \mathrm{~b}$ shows that $t_{3}=0$. 
Equation 22b simplifies to $t_{4} \bar{k}_{21}[\mathrm{M}]=t_{1} k_{21}[\mathrm{M}]$, from which we have $t_{1}=t_{4}$ and $\mathbf{T}=t_{1} \mathbf{I}_{2}$ ( $\mathbf{I}_{2}$ represents the $2 \times 2$ identity matrix). Equation 23 b shows that the alternative $\bar{b}_{2 i}$ is known up to a scaling factor.

The single-exponential $f(t)$ (eq 9) for this non-controllable system does not include $k_{01}$ and $k_{21}$. Hence, it is impossible to identify these rate constants. Once more, the identification via similarity transformation leads to incorrect conclusions for the noncontrollable photophysical system.

\section{D. Non-observable system with $a_{12}=0$ and $c_{2 j}=0$}

For the alternative $\overline{\mathbf{A}}$ of this non-observable photophysical system, see section 3.C. The components of the alternative $\overline{\mathbf{c}}_{j}$ (eq 17c) are

$$
\begin{aligned}
& \bar{c}_{1 j}=t_{1} c_{1 j} \\
& 0=t_{2} c_{1 j}
\end{aligned}
$$

From eq 24b, we have that $t_{2}=0$, so that from eq $24 \mathrm{a}$ we have that the alternative $\bar{c}_{1 j}$ is known up to a scaling factor $\left(t_{1}\right.$ must be different from zero to have an invertible $\mathbf{T}$ matrix).

The mono-exponential $f(t)$ (eq 11) for the non-observable system does not contain $k_{02}$; hence, it is impossible to identify $k_{02}$. Once again, these results are in disagreement with those from the identification via similarity transformation. 


\section{E. Non-controllable and non-observable systems with $[\mathrm{M}]$ given by eqs 13 and 15, respectively}

Now we investigate the identification via similarity transformation of the noncontrollable, intermolecular, two-state excited-state system with [M] given by eq 13 and of the corresponding non-observable system with [M] expressed by eq 15 . In Ref [10], we have studied the identifiability of the controllable and observable, intermolecular, two-state excited-state system via the similarity transformation approach. The identifiability analysis for the non-controllable system is formally equivalent to that described in Ref [10], but one has to exploit the required independence of $t_{i}$ of the excitation wavelength $\lambda_{i}^{\text {ex }}$ (i.e., through $b_{1 i}$ and $b_{2 i}$ ). For the non-observable system, one should use the necessary independence of $t_{i}$ of the observation wavelength $\lambda_{j}^{\mathrm{em}}$ (i.e., through $c_{1 j}$ and $c_{2 j}$ ). Since the identifiability analysis for the non-controllable or nonobservable systems of intermolecular two-state excited-state processes is very similar to that already published, ${ }^{10}$ we shall only present the results here. As before, two cases are found.

Case (i): When $t_{1}=t_{4}, t_{2}=t_{3}=0$, we have $\mathbf{T}=t_{1} \mathbf{I}_{2}$. The matrix multiplication in eq 17a with $\mathbf{T}=t_{1} \mathbf{I}_{2}$ gives $\bar{k}_{01}=k_{01}, \bar{k}_{21}=k_{21}, \bar{k}_{12}=k_{12}$, and $\bar{k}_{02}=k_{02}$. The corresponding matrix multiplication in eq $17 \mathrm{~b}$ with $\mathbf{T}=t_{1} \mathbf{I}_{2}$ for the non-controllable system gives $\bar{b}_{1 i}=b_{1 i} / t_{1}$ and $\bar{b}_{2 i}=b_{2 i} / t_{1}$. The analogous multiplication in eq $17 \mathrm{c}$ with $\mathbf{T}=t_{1} \mathbf{I}_{2}$ for the non-observable system gives $\bar{c}_{1 j}=c_{1 j} t_{1}$ and $\bar{c}_{2 j}=c_{2 j} t_{1}$. Hence, the alternative $\left\{\bar{b}_{1 i}, \bar{b}_{2 i}\right\}$ and $\left\{\bar{c}_{1 j}, \bar{c}_{2 j}\right\}$ are only known up to the scaling factor $t_{1}$. However, the pre-exponential factor in eqs 14 and 16 is uniquely defined: $\bar{b}_{1 i} \bar{c}_{1 j}+\bar{b}_{2 i} \bar{c}_{2 j}=b_{1 i} c_{1 j}+b_{2 i} c_{2 j}$. 
Case (ii): Alternatively, we have $\bar{k}_{01}=k_{02}+k_{12}, \bar{k}_{21}=k_{21}, \bar{k}_{12}=k_{01}-k_{02}$, and $\bar{k}_{02}=k_{02}$. The matrix $\mathbf{T}$ takes the form

$$
\mathbf{T}=\left(\begin{array}{cc}
t_{1} & 0 \\
t_{3} & t_{1}+t_{3}
\end{array}\right)
$$

Yet again, the identification via similarity transformation indicates that for the noncontrollable and non-observable, intermolecular, two-state excited-state systems more distinct parameters could be identified than is possible from the mono-exponential $f(t)=$ $\alpha \exp (\gamma t)$ (eqs 14 and 16). Indeed, for the non-controllable system, it is impossible to determine the separate model parameters from $\gamma=-\left(b_{1 i} k_{01}+b_{2 i} k_{02}\right) /\left(b_{1 i}+b_{2 i}\right)$ (see eq 14$)$. Similarly, for the non-observable system, the individual model parameters cannot be determined from $\gamma=-\left[c_{2 j}\left(k_{02}+k_{12}\right)-c_{1 j} k_{12}\right] / c_{2 j}$ (see eq 16).

\section{Discussion and conclusions}

In the deterministic identifiability analysis, the central question is whether it is possible to find a unique solution for each of the unknown parameters of the proposed model, assuming perfect (i.e., without noise) data. Identifiability is also of importance in the

design of time-resolved fluorescence experiments (choice of excitation, $\lambda_{i}^{\text {ex }}$, and emission, $\lambda_{j}^{\mathrm{em}}$, wavelengths; the number and the nature of the used co-reactant concentrations; the need to add quencher to the photophysical system; etc.) that lead to unique solutions. A number of alternative approaches to identifiability analysis are available and have been employed for the analysis of the identification of common photophysical models. We have extensively used Markov parameters and elementary functions of the rate constants for this purpose (see literature references in [9] and [10]). 
This identifiability method is closely related to the Laplace transform approach (or transfer function approach). ${ }^{4}$ The Taylor series expansion of the fluorescence $\delta$-response function $f(t)$ is an alternative method, which was used in the identifiability of fluorescence quenching of stationary probes by mobile quencher molecules in micelles. ${ }^{17}$ The advantage of the Taylor series expansion of $f(t)$ is its applicability to nonlinear models.

Similarity transformation is a powerful identifiability technique because it offers an excellent method of finding an alternative realization $\left(\overline{\mathbf{A}}, \overline{\mathbf{b}}_{i}, \overline{\mathbf{c}}_{j}\right)$ of $f(t)$ and of determining whether the model is uniquely or locally identifiable or not identifiable at all. In time-resolved fluorescence, a model is uniquely identifiable if all parameters can be uniquely determined from $f(t)$. If there are several parameter values that correspond to exactly the same $f(t)$, the model is locally identifiable. An unidentifiable model is found when there is an unlimited number of alternative model parameter values corresponding to the same $f(t)$. In the latter case it is problematic to attempt to estimate the parameter values, because the solution found is only one of many and has no reason to be favored. An extra bonus of the similarity transformation approach is that the relationships between the true and alternative model parameters are explicitly provided by eqs $17 \mathrm{a}-\mathrm{c}$. However, the disadvantage of this identifiability technique is that one should not apply it when the conditions for its validity do not hold (i.e., both controllability and observability). ${ }^{7}$ One can convincingly argue that - since there are several other identification methods - one can always use a quite straightforward technique (e.g., transfer function approach) for which controllability and observability are not an issue. 
There is, however, another important feature to controllability and observability in photophysics without direct relation to identifiability methods. Indeed, non-controllable or non-observable systems are found whenever the rank of the matrices $\mathbf{R}$ and $\mathbf{O}$, respectively, is reduced. For the intermolecular, two-state excited-state system studied, $\operatorname{rank} \mathbf{R}=1$ for the non-controllable system and $\operatorname{rank} \mathbf{O}=1$ for the non-observable system. For the controllable and observable photophysical system, one always observes two exponential terms in $f(t)$ representing the two eigenvalues of matrix $\mathbf{A}$ (eq 3 ), whereas the non-controllable or non-observable system always leads to single-exponential $f(t)$ at all observation wavelengths $\lambda_{j}^{\mathrm{em}}$ or at all excitation wavelengths $\lambda_{i}^{\mathrm{ex}}$, respectively. If one observes a mono-exponential $f(t)$, while expecting a bi-exponential $f(t)$, controllability and observability are useful, systematic tools to explore the reason for this: either a ground-state species is not excited or an excited species is not observed in addition to some zero-value exchange rate constants. The two next paragraphs summarize the possible causes for the "pathological", mono-exponential responses $f(t)$ for an intermolecular two-state excited-state system.

For intermolecular two-state excited-state processes, we have a non-controllable system under three conditions (see sections 3.A, 3.C, and 3.E). In these three cases, $f(t)$ remains mono-exponential, whatever the emission wavelength $\lambda_{j}^{\mathrm{em}}$. For intermolecular two-state excited-state processes, one usually measures fluorescence traces $d(t)$ as a function of coreactant concentration $[\mathrm{M}]$, excitation wavelength $\lambda_{i}^{\mathrm{ex}}$, and emission wavelength $\lambda_{j}^{\mathrm{em}}$, and one extracts the relevant decay parameters of $f(t)$ from the resulting fluorescence decay surface via a global nonlinear fitting. (a) If a single-exponential $f(t)$ is found for $[\mathrm{M}]=0$ for all observation wavelengths $\lambda_{j}^{\mathrm{em}}$, this implies that only ground-state species $\mathbf{1}$ 
is being excited $\left(b_{2 i}=0\right)$. (b) A single-exponential $f(t)$ found for all $[\mathrm{M}]$ and at all $\lambda_{j}^{\mathrm{em}}$ indicates that either $k_{12}=0$ and only ground-state species 2 is being excited $\left(b_{1 i}=0\right.$, Figure $2 \mathrm{~b}$, eq 9$)$ or $k_{21}=0$ and only ground-state species $\mathbf{1}$ is being excited $\left(b_{2 i}=0\right.$, Figure 2a, eq 8). In both cases, the single-exponential $f(t)$ is independent of [M]. Absorption spectra of $\mathbf{1}$ and/or $\mathbf{2}$ can possibly be used to make a distinction between the two cases. (c) When a mono-exponential $f(t)$ is observed at a single, non-zero [M] and at all $\lambda_{j}^{\mathrm{em}}$, then that particular $[\mathrm{M}]$ is given by eq 13 and $f(t)$ by eq 14 . At $[\mathrm{M}]$ values different from that given by eq $13, f(t)$ is bi-exponential.

For intermolecular two-state excited-state processes, a non-observable system is found under three conditions (see sections 3.B, 3.D, and 3.E). These three cases result in monoexponential $f(t)$, independent of the excitation wavelength $\lambda_{i}^{\text {ex }}$. (a) A single-exponential $f(t)$ at $[\mathrm{M}]=0$ and at all $\lambda_{i}^{\text {ex }}$ means that the fluorescence of only excited species $\mathbf{2}^{*}$ is being observed $\left(c_{1 j}=0\right)$. (b) A single-exponential $f(t)$ found for all $[\mathrm{M}]$ and at all $\lambda_{i}^{\mathrm{ex}}$ indicates either that $k_{12}=0$ and the fluorescence of exclusively excited species $\mathbf{1}^{*}$ is being monitored $\left(c_{2 j}=0\right.$, Figure 3a, eq 11) or $k_{21}=0$ and only the fluorescence of $\mathbf{2}^{*}$ is being observed $\left(c_{1 j}=0\right.$, Figure $3 b$, eq 12$)$. In the former case, the fluorescence $\delta$-response function $f(t)$ depends on $[\mathrm{M}]$ (eq 11), while in the latter case $f(t)$ is independent of $[\mathrm{M}]$ (eq 12). (c) When a mono-exponential $f(t)$ is observed at a single, "critical", non-zero [M] and at all $\lambda_{i}^{\text {ex }}$, this $[\mathrm{M}]$ is given by eq 15 and $f(t)$ by eq 16 . At $[\mathrm{M}]$ values different from that given by eq $15, f(t)$ is bi-exponential.

To summarize, single-exponential fluorescence decays observed for intermolecular twostate excited-state processes are always the consequence of non-controllability or non- 
observability of the photophysical system. For controllable and observable systems, fitting of the experimental fluorescence decay data requires the same number of distinct exponential terms as the number of excited species. Conversely, if the number of exponential terms in $f(t)$ is less than the number of excited species, the photophysical system is not controllable or not observable. The importance of controllability and observability to the field of photophysics lies in the fact that these concepts allow one to systematically investigate the origins of the reduced number of exponential terms in $f(t)$, and to extend the study to more complicated photophysical systems than that considered here.

\section{Figure captions}

Figure 1. Kinetic model of an intermolecular, two-state excited-state process. The excited-state processes are described by the deactivation rate constants $k_{01}$ and $k_{02}$, and the excited-state exchange rate constants $k_{21}$ and $k_{12}$. The transformation of species 1 and $\mathbf{1}^{*}$ into, respectively, 2 and $\mathbf{2}^{*}$ is mediated by co-reactant M. Photoexcitation at $\lambda_{i}^{\text {ex }}$ is symbolized by $u(t)$.

Figure 2. Non-controllable photophysical systems. (a) $b_{2 i}=0$ and $a_{21}=0$. (b) $b_{1 i}=0$ and $a_{12}=0$. Photoexcitation at $\lambda_{i}^{\text {ex }}$ is symbolized by $u(t)$.

Figure 3. Non-observable photophysical systems. (a) $c_{2 j}=0$ and $a_{12}=0$. (b) $c_{1 j}=0$ and $a_{21}=0$. Observation at $\lambda_{j}^{\mathrm{em}}$ of the fluorescence from a specific, excited species is depicted by an eye on top of that excited species. 


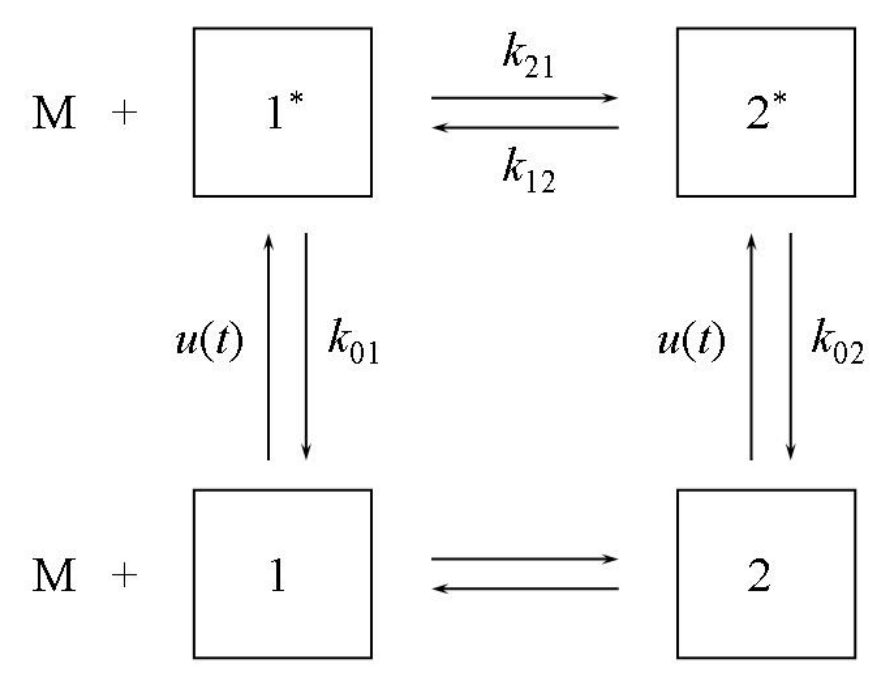

Fig. $1 \uparrow$

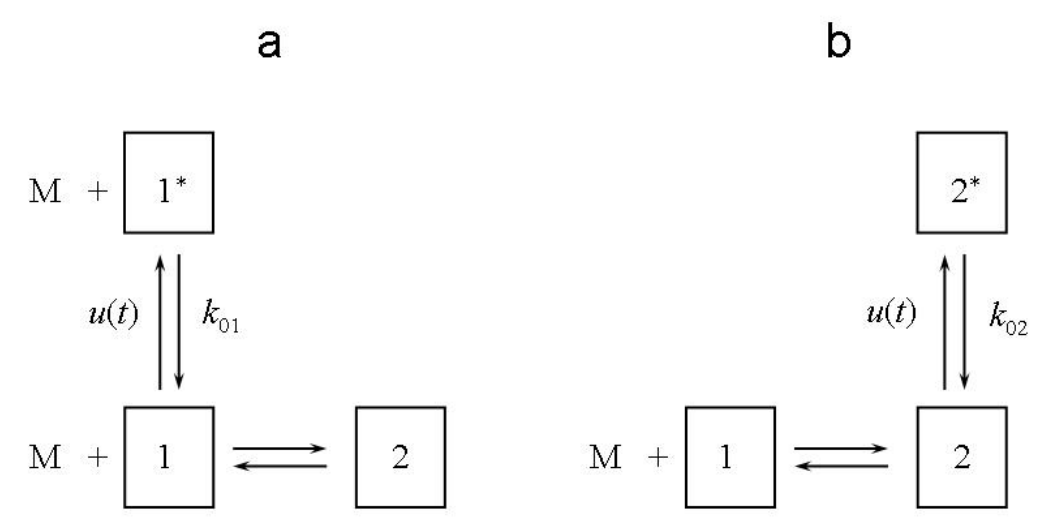

Fig. $2 \uparrow$ 


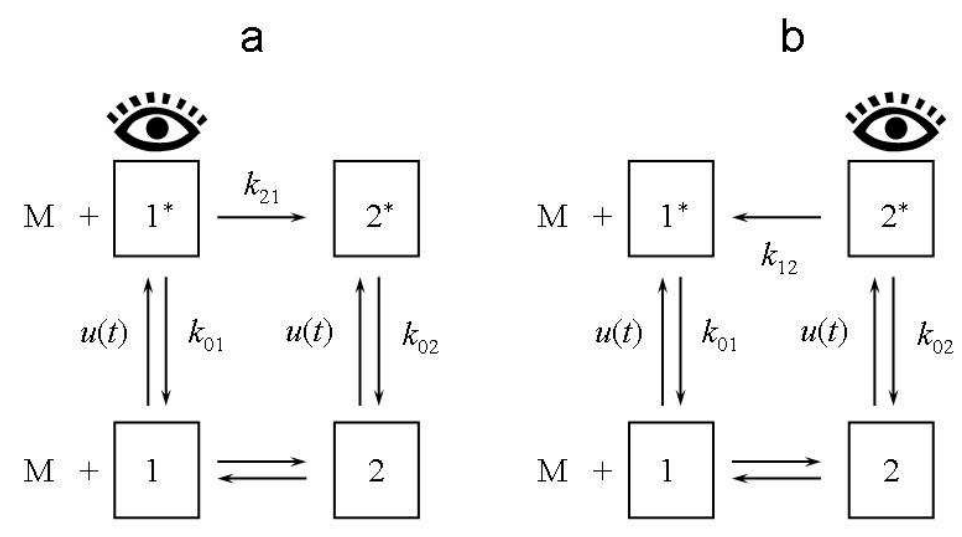

Fig. $3 \uparrow$

\section{References}

[1] O'Connor, D. V.; Phillips, D. Time-Correlated Single Photon Counting, Academic Press: London, 1984.

[2] Boens, N.; Qin, W. ; Basarić, N.; Hofkens, J.; Ameloot, M.; Pouget, J.; Lefèvre, J.-P.; Valeur, B.;

Gratton, E.; vandeVen, M.; Silva, N. D.; Engelborghs, Y.; Willaert, K.; Sillen, A.; Rumbles, G.;

Phillips, D.; Visser, A. J. W. G.; Van Hoek, A.; Lakowicz, J. R.; Malak, H.; Gryczynski, I.; Szabo, A.

G.; Krajcarski, D. T.; Tamai, N.; Miura, A. Anal. Chem. 2007, 79, 2137-2149.

[3] vandeVen, M.; Ameloot, M.; Valeur, B.; Boens, N. J. Fluorescence 2005, 15, 377-413.

[4] Godfrey, K. Compartmental Models and Their Application, Academic Press: New York, 1983.

[5] Anderson, D. H. Compartmental Modeling and Tracer Kinetics, in Lecture Notes in Biomathematics, vol. 50, Springer-Verlag: Berlin, 1983.

[6] Jacquez, J. A. Compartmental Analysis in Biology and Medicine, 3rd Ed., BioMedware: Ann Arbor, 1996. 
[7] Walter, E. Identifiability of Parametric Models, Pergamon Press: Oxford, 1987.

[8] Ameloot, M.; Beechem, J. M.; Brand, L. Chem. Phys. Lett. 1986, 129, 211-219.

[9] Boens, N.; Ameloot, M. Int. J. Quantum Chem. 2006, 106, 300-315.

[10] Boens, N.; Szubiakowski, J.; Novikov, E.; Ameloot, M. J. Chem. Phys. 2000, 112, 8260-8266.

[11] Szubiakowski, J. P.; Dale, R. E.; Boens, N.; Ameloot, M. J. Chem. Phys. 2004, 121, 7829-7839.

[12] Boens, N.; Novikov, E.; Ameloot, M. J. Phys. Chem. A 2005, 109, 7024-7032.

[13] Boens, N.; Novikov, E.; Szubiakowski, J. P.; Ameloot, M. J. Phys. Chem. A 2005, 109, 11655-11664.

[14] Boens, N.; Novikov, E.; Ameloot, M. ChemPhysChem 2006, 7, 2559-2566.

[15] Szubiakowski, J. P.; Dale, R. E.; Boens, N.; Ameloot, M. Chem. Phys. Lett. 2007, 438, 113-118.

[16] Boens, N.; Van der Auweraer, M. ChemPhysChem 2005, 6, 2353-2358.

[17] Boens, N.; Novikov, E.; Van der Auweraer, M. Math. Biosci. 2007, 209, 624-643.

[18] Eisenfeld, J.; Ford, C. C. Biophys. J. 1979, 26, 73-84.

[19] Ameloot, M.; Boens, N.; Andriessen, R.; Van den Bergh, V.; De Schryver, F. C. J. Phys. Chem. 1991, $95,2041-2047$.

[20] Sontag, E. D. Mathematical Control Theory: Deterministic Finite Dimensional Systems. 2nd Ed., Springer, New York, 1998.

[21] Kalman, R. E.; Falb, P. L.; Arbib, M. A. Mathematical System Theory, McGraw-Hill: New York, 1969. 